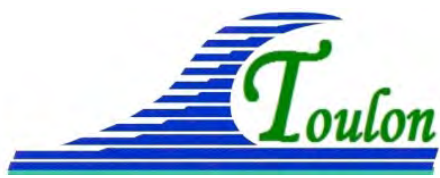

XIV èmes Journées Nationales Génie Côtier - Génie Civil Toulon, 29 juin au $1^{\text {er }}$ juillet 2016

DOI:10.5150/jngcgc.2016.012 (C) Editions Paralia CFL disponible en ligne - http://www.paralia.fr - available online

\title{
Développement d'un modèle numérique non-linéaire et dispersif pour la propagation des vagues en zone côtière
}

\author{
Cécile RAOULT $^{1,2}$, Michel BENOIT ${ }^{3}$, Marissa L. YATES ${ }^{1,4}$
}

1. Laboratoire d'Hydraulique Saint-Venant, Université Paris-Est, (unité de recherche commune EDF R\&D, Cerema, Ecole de Ponts ParisTech), 6 quai Watier, BP 49, F-78401 Chatou cedex, France.

2. EDF R\&D, Laboratoire National d'Hydraulique et Environnement (LNHE) 6 quai Watier, BP 49, F-78401 Chatou cedex, France.

3. Institut de Recherche sur les Phénomènes Hors-Equilibre (IRPHE), UMR 7342 (CNRS, Aix-Marseille Université, Ecole Centrale Marseille), 49 rue Frédéric JoliotCurie, BP 146, F-13384 Marseille Cedex 13, France

4. Cerema, Division technique Eau, mer et fleuves

134 rue de Beauvais, F-60280 Margny-les-Compiègne, France.

cecile.raoult@edf.fr ; benoit@irphe.univ-mrs.fr ; marissa.yates-michelin@cerema.fr

\section{Résumé :}

Les effets non-linéaires et dispersifs étant particulièrement importants pour les vagues en zone côtière, nous étudions et développons un modèle potentiel complètement nonlinéaire et dispersif résolvant les équations d'Euler-Zakharov qui régissent l'évolution temporelle de la position et du potentiel des vitesses à la surface libre.

La formulation mathématique ainsi que sa mise en œuvre numérique sont rappelées, avec la présentation de la méthode d'extension du domaine d'une à deux dimensions d'espace horizontales. Les capacités non-linéaires et dispersives de la version 1DH du modèle sont démontrées à travers l'application à deux cas tests : d'abord, la génération et la propagation des harmoniques libres et liées associées aux vagues régulières créées par un générateur de vagues de type piston sur un fond plat d'après les expériences de CHAPALAIN et al. (1992), puis la propagation de vagues irrégulières au-dessus d'une barre sous-marine d'après les expériences de BECQ-GIRARD et al. (1999). La bonne représentation des transferts d'énergie entre les différentes composantes harmoniques montre la capacité et la précision du modèle à représenter les effets dispersifs et non linéaires d'ordres élevés. Le développement d'une version 2DH du modèle a été testé pour simuler la propagation de vagues régulières sur une marche immergée semicirculaire agissant comme une lentille convergente, afin de reproduire une des expériences de WHALIN (1971). Les bons résultats obtenus sont encourageants quant à l'utilisation des fonctions de base radiales pour traiter des cas de bathymétries irrégulières réalistes en $(x, y)$.

Mots-clés : Vagues non-linéaires, Hydrodynamique côtière, Simulation des vagues, Modélisation numérique, Modèles de vagues, Fonctions de base radiales. 


\section{Introduction}

La transformation des vagues depuis le large jusqu'à la plage fait intervenir un grand nombre de processus physiques, incluant la réfraction et le levage (shoaling), des effets dissipatifs dus à la friction sur le fond ou au déferlement, la diffraction et la réflexion sur des structures, îles ou hauts-fonds, etc. La modélisation précise de l'ensemble de ces processus est loin d'être triviale, compte tenu notamment du fait que la profondeur d'eau relative pour les vagues $\mu=k h$ (où $k$ est le nombre d'onde et $h$ la profondeur d'eau) varie très significativement entre le large et la plage. Par ailleurs, les effets nonlinéaires, quantifiés à l'aide du paramètre de cambrure $\varepsilon=k H / 2$ ou de hauteur de vague relative $H / h$ (où $H$ est la hauteur caractéristique des vagues), peuvent devenir importants en zone côtière avec la diminution de la profondeur d'eau.

Pour traiter correctement ces deux aspects, les modèles numériques de simulation des vagues doivent posséder des capacités dispersives et non-linéaires élevées. Certaines approches de modélisation utilisées couramment en ingénierie côtière et portuaire, fondées par exemple sur l'équation de pente douce (mild slope equation) de BERKHOFF (1972) dans sa version originale ou ses extensions, font le choix de négliger les effets non-linéaires pour la propagation des vagues. Les modèles de type Boussinesq, Serre ou Green-Naghdi permettent une prise en compte partielle des effets non-linéaires et/ou dispersifs (e.g. MADSEN et al., 1991 ; NWOGU, 1993 ; KIRBY, 2003 ; CHAZEL et al., 2011). Cependant, ces modèles ne sont que partiellement dispersifs et/ou partiellement non-linéaires, même dans le cas de développements d'ordres élevés (MADSEN et al., 2002, 2006 ; BENOIT \& CHAZEL, 2013).

Dans le but de disposer d'un outil de simulation précis, les auteurs ont choisi de développer un modèle complètement dispersif et non-linéaire, fondé sur les équations surfaciques d'Euler-Zakharov (ZAKHAROV, 1968) dans un cadre potentiel (YATES \& BENOIT, 2015). Le code de simulation numérique, appelé Misthyc, est développé en collaboration entre l'IRPHE et le Laboratoire Saint-Venant. RAOULT et al. (2014) ont présenté une première série de cas de validation de sa version $1 \mathrm{DH}(x, z)$. Ici, les avancées récentes sur le développement et la validation de ce modèle numérique sont présentées.

Nous rappelons brièvement les fondements mathématiques du modèle dans la partie 2, puis donnons dans la partie 3 un aperçu des méthodes numériques utilisées. Dans la partie 4, deux cas de validation 1DH mettant en jeu des effets non-linéaires importants sont présentés et analysés en détail. Une première application sur un cas 2DH (i.e. 3D) est présentée en partie 5 avec la simulation d'une des expériences de WHALIN (1971) sur une bathymétrie provoquant une convergence des vagues. Les conclusions et perspectives sont résumées dans la partie 6. 


\section{XIV ${ }^{\text {èmes }}$ Journées Nationales Génie Côtier - Génie Civil \\ Toulon, 29 juin au $1^{\text {er }}$ juillet 2016}

\section{Présentation du modèle mathématique}

En considérant l'écoulement irrotationnel d'un fluide homogène, non visqueux et de masse volumique constante, une modélisation mathématique potentielle est adoptée. Le système est décrit par le potentiel des vitesses $\Phi(\vec{x}, z, t)$, qui vérifie l'équation de Laplace dans tout le domaine fluide, avec la notation $\vec{x}=(x, y)$. Cette équation est complétée par deux conditions aux limites (non-linéaires), dynamique et cinématique, à la surface libre en $z=\eta(\vec{x}, t)$, une condition d'imperméabilité au fond en $z=-h(\vec{x}, t)$ et des conditions aux frontières latérales de type Dirichlet ou Neumann. En supposant la continuité de la colonne d'eau entre le fond et la surface, on peut définir le potentiel des vitesses à la surface libre $\Psi(\vec{x}, t)=\Phi(\vec{x}, z=\eta(\vec{x}, t), t)$ et les conditions aux limites à la surface libre deviennent (ZAKHAROV, 1968) :

$\frac{\partial \eta}{\partial t}=-\nabla \eta \cdot \nabla \Psi+\tilde{w}\left(1+(\nabla \eta)^{2}\right)$

$\frac{\partial \Psi}{\partial t}=-g \eta-\frac{1}{2}(\nabla \Psi)^{2}+\frac{1}{2} \widetilde{w}^{2}\left(1+(\nabla \eta)^{2}\right)$

où $\widetilde{w}(\vec{x}, t) \equiv \frac{\partial \Phi}{\partial z}(\vec{x}, z=\eta, t)$ est la composante verticale de la vitesse en surface libre.

Le système (1-2) ne fait intervenir que des variables surfaciques. Cependant, l'intégration en temps de ces équations nécessite de connaître $\widetilde{w}$, qui doit être estimée à partir des deux quantités $\eta$ et $\psi$, problème appelé "Dirichlet-to-Neumann" (DtN).

\section{Présentation de la résolution numérique}

La simulation numérique du système (1-2) nécessite : un schéma d'intégration en temps, une méthode de résolution du problème $\mathrm{DtN}$ et une méthode de calcul des dérivées premières et secondes en espace. Les détails des méthodes numériques pour la version 1DH $(x, z)$ du modèle sont disponibles dans YATES \& BENOIT (2015) et RAOULT et al. (2014); seuls les points principaux sont rappelés ici. Pour l'avance en temps, un schéma de Runge-Kutta explicite d'ordre 4, avec un pas de temps constant, est utilisé. Le problème DtN est résolu en utilisant une méthode spectrale sur la verticale (TIAN \& SATO, 2008) pour trouver la solution du problème aux limites de Laplace pour le potentiel $\Phi$ dans tout le domaine. Une base de polynômes de Tchebychev de première espèce est utilisée jusqu'à l'ordre $N_{T}$ (valant 5 à 10 pour les applications pratiques). Dans la version 1DH du modèle, des schémas aux différences finies d'ordre 4 sont utilisés pour calculer les dérivées spatiales, avec un pas d'espace variable dans le cas général.

Afin d'aborder des cas plus réalistes, avec des configurations bathymétriques complexes et des vagues d'incidences multiples, le modèle a été étendu à deux dimensions horizontales (i.e. en 3D) en utilisant une méthode de fonctions de base radiales (RBF) (WRIGHT \& FORNBERG, 2006) sur une discrétisation par nuage de points dans le plan $(x, y)$ afin de s'adapter facilement à tout type de géométrie, tout en gardant une 
représentation spectrale sur la verticale. On cherche à approximer la dérivée d'une fonction $f$ en un nœud $\overrightarrow{x_{l}}$ comme une combinaison linéaire des valeurs de la fonction $f$ au $n-1$ plus proches voisins ( $n$ étant le nombre de points dans la cellule ou "stencil") :

$L(f)\left(\overrightarrow{x_{l}}\right)=\sum_{k=1}^{n} w_{i, k}^{L} f\left(\overrightarrow{x_{k}}\right)$

Avec $L$ un opérateur différentiel linéaire quelconque et les $w_{i, k}^{L}$ les inconnues à déterminer pour chaque opérateur $L$ et chaque nœud $\overrightarrow{x_{l}}$. Pour cela on utilise un ensemble de fonctions tests formant une base, pour lesquelles on impose que l'approximation soit exacte. Ici on choisit des fonctions radiales, centrées aux nœuds du stencil $\overrightarrow{x_{k}}, k=1, n$ : $\varphi_{k}(\vec{x})=\Phi\left(\left\|\vec{x}-\overrightarrow{x_{k}}\right\|\right)$. Cela conduit à résoudre un système linéaire de $n$ équations à $n$ inconnues dont la formulation matricielle est la suivante :

$\left[\begin{array}{cccc}\Phi\left(\left\|\overrightarrow{x_{1}}-\overrightarrow{x_{1}}\right\|\right) & \Phi\left(\left\|\overrightarrow{x_{2}}-\overrightarrow{x_{1}}\right\|\right) & \ldots & \Phi\left(\left\|\overrightarrow{x_{n}}-\overrightarrow{x_{1}}\right\|\right) \\ \Phi\left(\left\|\overrightarrow{x_{1}}-\overrightarrow{x_{2}}\right\|\right) & \Phi\left(\left\|\overrightarrow{x_{2}}-\overrightarrow{x_{2}}\right\|\right) & & \vdots \\ \vdots & \vdots & \vdots \\ \Phi\left(\left\|\overrightarrow{x_{1}}-\overrightarrow{x_{n}}\right\|\right) & \ldots & \ldots & \Phi\left(\left\|\overrightarrow{x_{n}}-\overrightarrow{x_{n}}\right\|\right)\end{array}\right]\left[\begin{array}{c}w_{i, 1}^{L} \\ w_{i, 2}^{L} \\ \vdots \\ w_{i, n}^{L}\end{array}\right]=\left[\begin{array}{c}L \Phi\left(\left\|\vec{x}-\overrightarrow{x_{1}}\right\|\right)\left(\overrightarrow{x_{l}}\right) \\ L \Phi\left(\left\|\vec{x}-\overrightarrow{x_{2}}\right\|\right)\left(\overrightarrow{x_{l}}\right) \\ \vdots \\ L \Phi\left(\left\|\vec{x}-\overrightarrow{x_{n}}\right\|\right)\left(\overrightarrow{x_{l}}\right)\end{array}\right]$

Avec des nœuds fixes, ce système est résolu, une seule fois en début de simulation, pour chacun des nœuds du domaine et pour chaque opérateur différentiel. Par la suite, la fonction radiale utilisée est la fonction multiquadrique (WRIGHT \& FORNBERG, 2006) :

$\Phi\left(\left\|\vec{x}-\overrightarrow{x_{k}}\right\|\right)=\sqrt{\left\|\vec{x}-\overline{x_{k}}\right\|^{2}+c^{2}}$

où $c$ est le paramètre de forme. Plus la valeur de $c$ est élevée plus la multiquadrique est aplatie, ce qui a pour effet de diminuer l'erreur d'approximation, mais en contrepartie dégrade le conditionnement de la matrice et donc augmente la difficulté de résolution du système linéaire et les erreurs d’arrondi.

\section{Validations de la version 1DH du modèle numérique}

\subsection{Dynamique d'un train de vagues généré par un batteur piston sur fond plat}

CHAPALAIN et al. (1992) ont étudié la propagation de vagues générées par le mouvement sinusoïdal d'un batteur piston sur un fond plat. Ce cas test consiste à simuler l'essai A correspondant à un piston se déplaçant d'une amplitude maximale de $e=7,8 \mathrm{~cm}$, à une période de $T=2,5 \mathrm{~s}$, dans une profondeur d'eau de $h=0,4 \mathrm{~m}$. D'après la relation de dispersion linéaire des vagues, on peut estimer la longueur d'onde des vagues générées à $L=4,74 \mathrm{~m}$. Pour la simulation numérique, on impose à la frontière gauche du modèle (côté batteur), une vitesse homogène sur la verticale, avec une variation sinusoïdale en temps. La frontière droite est suffisamment loin du batteur pour négliger les réflexions. On laisse les vagues se propager pendant 16 périodes (soit $40 \mathrm{~s}$ ) avec un pas de temps $\Delta t=T / 40=0,0625 \mathrm{~s}$. L'ordre maximal des polynômes de Tchebychev est fixé à $N_{T}=7$ et on utilise un maillage régulier en $x$, avec $\Delta x=0,1 \mathrm{~m}$. 


\section{XIV ${ }^{\text {èmes }}$ Journées Nationales Génie Côtier - Génie Civil \\ Toulon, 29 juin au $1^{\text {er }}$ juillet 2016}

Une fois le régime stationnaire atteint, une analyse harmonique du signal temporel d'élévation de surface libre évalue les amplitudes et les phases des différentes harmoniques (Figure 1a). Le modèle représente correctement le transfert d'énergie entre les différentes harmoniques, avec les bonnes longueurs de battement, due la présence simultanée de composantes libres et liées des harmoniques à 2, 3 et 4 fois la fréquence de mouvement du batteur. L'écart observé pour la deuxième harmonique (2f) après $x=19$ $\mathrm{m}$, dont l'amplitude diminue légèrement dans les expériences, est probablement dû aux effets dissipatifs qui ne sont pas pris en compte par le modèle. L'évolution spatiale de la différence de phase entre la première et la seconde harmonique (Figure 1b), oscillant entre $-\pi / 2$ et $+\pi / 2$ avec la même longueur de battement que les amplitudes, est également bien reproduite par le modèle. Les deux premières harmoniques sont en phase quand l'amplitude de la première harmonique est maximale et celle de la seconde minimale, et vice versa.

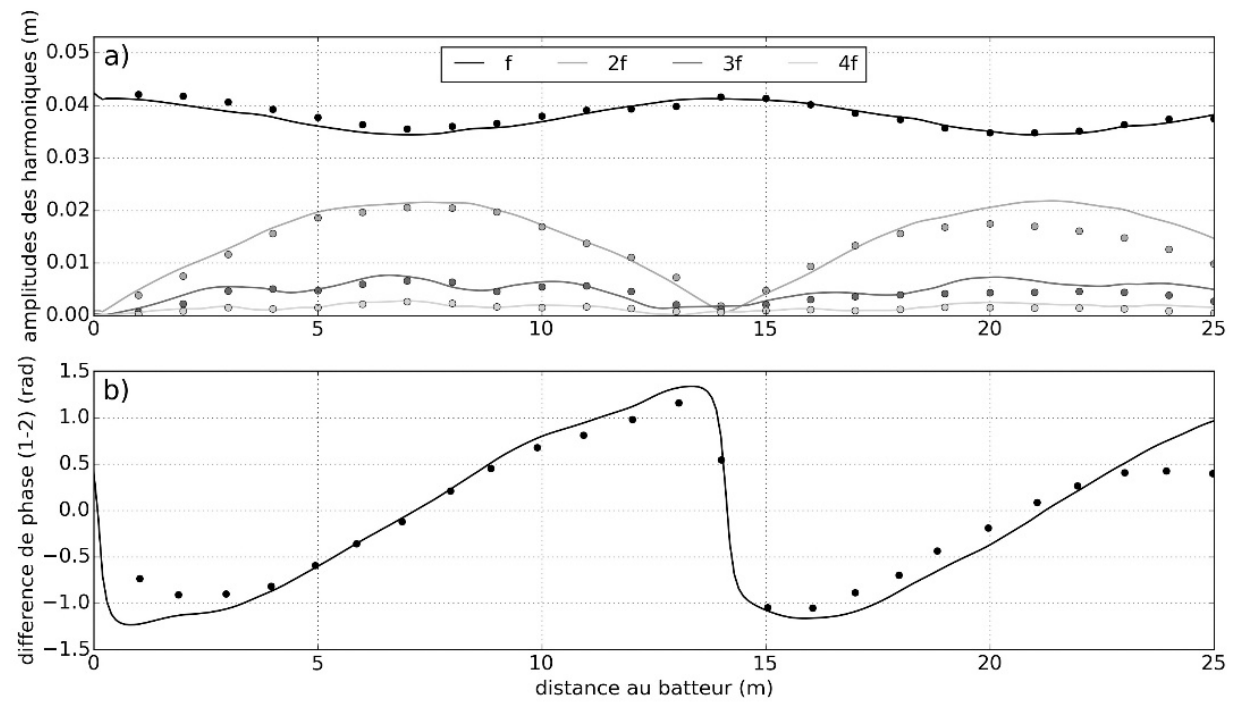

Figure 1. a) Evolution spatiale des amplitudes des quatre premières harmoniques d'élévation de surface libre pour le cas A de CHAPALAIN et al. (1992). b) Evolution spatiale de la différence de phase entre la première et la seconde harmonique. Les résultats expérimentaux sont représentés par les points et les résultats des simulations par les lignes continues.

\subsection{Propagation de vagues irrégulières sur une barre sous-marine}

Ce cas test a pour but de simuler la propagation de vagues irrégulières au-dessus d'une barre sous-marine d'après les expériences de BECQ-GIRARD et al. (1999). Le profil bathymétrique (Figure 2) a été spécialement conçu afin d'étudier les effets non-linéaires en eau peu profonde. Expérimentalement, une série de vagues irrégulières est générée à l'aide d'un piston selon un spectre JONSWAP avec un facteur d'élancement $\gamma=3,3$, de période pic $T_{p}=2,39 \mathrm{~s}$ et de hauteur significative $H_{m 0}=3,4 \mathrm{~cm}$ par $0,65 \mathrm{~m}$ de fond au 
large du profil bathymétrique (essai 26 de BECQ-GIRARD et al. (1999) sans déferlement sur la barre sous-marine). L'élévation de surface libre est mesurée par 16 sondes pendant $40 \mathrm{~min}$ avec un pas d'échantillonnage de $\Delta t=0,07 \mathrm{~s}$.

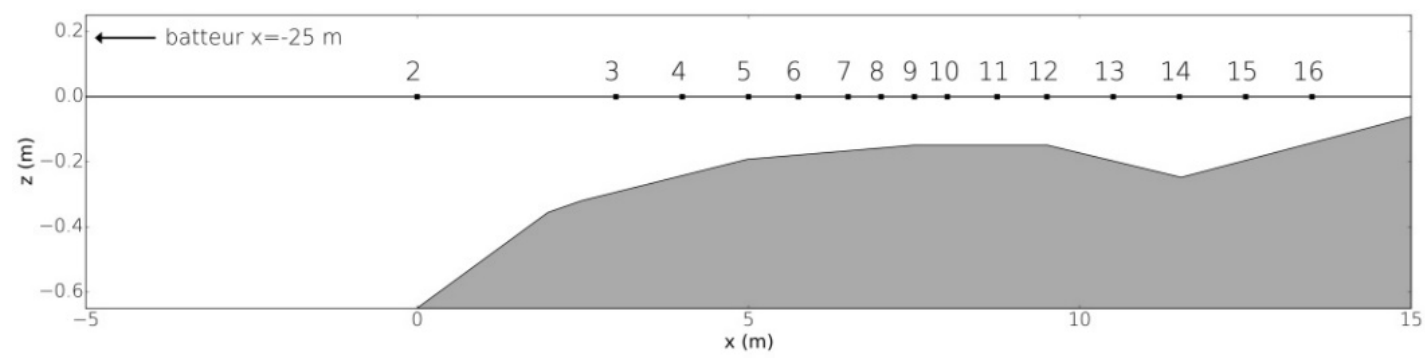

Figure 2. Bathymétrie et positions des sondes de mesure de vagues pour les expériences de BECQ-GIRARD et al. (1999).

Dans la simulation, le domaine de calcul s'étend de $x=-5 \mathrm{~m}$ à $+25 \mathrm{~m}$, le début de la barre se situant à $x=0 \mathrm{~m}$; il est discrétisé de manière régulière avec un pas d'espace $\Delta x=0,05 \mathrm{~m}$, et $N_{T}=7$. Pour forcer le modèle sur la limite côté batteur, on utilise la série temporelle de surface libre mesurée à la sonde 2. Le potentiel associé est reconstruit à partir d'une analyse spectrale de cette surface libre en utilisant la théorie linéaire des vagues, et imposé sur la frontière gauche du domaine. Une zone de relaxation de $5 \mathrm{~m}$ de large est ajoutée pour la génération dans laquelle une correction de la position de la surface libre et du potentiel des vitesses est appliquée. De l'autre côté du domaine, une zone de relaxation de $10 \mathrm{~m}$ de large est également ajoutée pour absorber les vagues. La durée simulée est de 2380 s, soit environ 39,7 min avec un pas de temps identique à celui d'échantillonnage des sondes $\Delta t=0,07 \mathrm{~s}$.

Les spectres de variance de la surface libre montrent un bon accord avec les mesures (e.g. Figure 3, sondes 2, 9, 15 et 16). On observe en particulier un transfert d'énergie qui s'effectue du pic principal (à la fréquence $f_{p}$ ) vers les hautes fréquences avec l'apparition de pics dans le spectre de la sonde 9 aux fréquences harmoniques ( $2 f_{p}, 3 f_{p}$, etc.) et une bonne représentation des amplitudes des pics même pour la cinquième harmonique. Juste après la barre, le transfert d'énergie s'inverse, avec un transfert principalement dirigé vers la seconde harmonique. On remarque bien, dans le spectre de la sonde 15, la quasi-disparition des pics aux harmoniques 3, 4 et 5 ainsi que l'augmentation du second pic. Finalement, dans le spectre de la sonde 16, le pic de la troisième harmonique est de nouveau bien marqué du fait de la diminution de la profondeur d'eau sur la partie terminale du profil bathymétrique. 


\section{XIVìmes Journées Nationales Génie Côtier - Génie Civil \\ Toulon, 29 juin au $1^{\text {er }}$ juillet 2016}
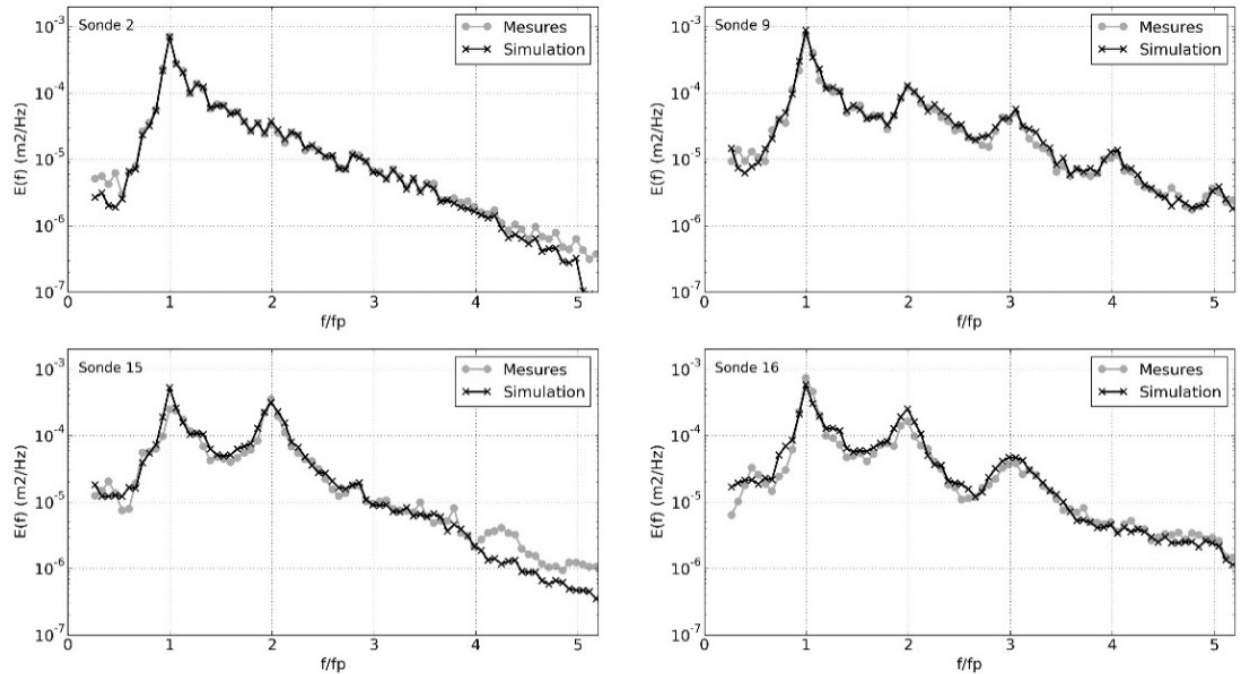

Figure 3. Comparaison des spectres de variance de la surface libre mesurés (en gris) et simulés (en noir) à certaines sondes (2, 9, 15 et 16) pour l'essai 26 de BECQ-GIRARD et al. (1999).
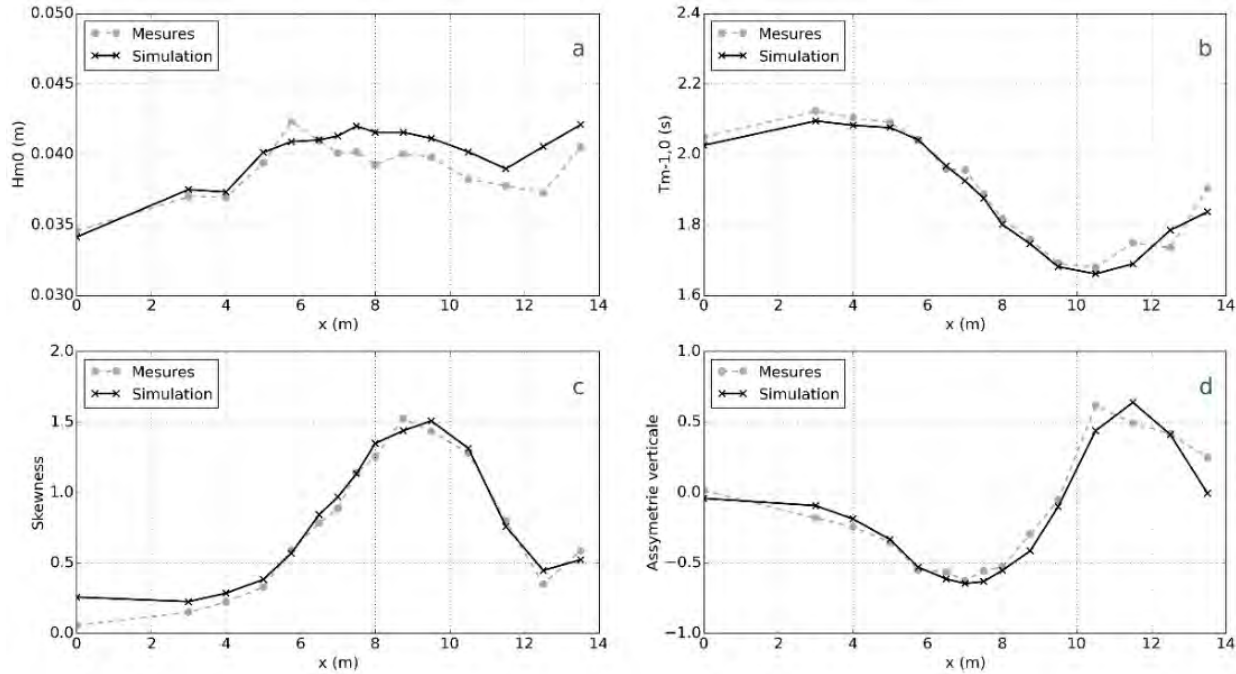

Figure 4. Comparaison des paramètres d'état de mer mesurés (en gris) et simulés (en noir) le long du profil bathymétrique des expériences de BECQ-GIRARD et al. (1999) : a) hauteur significative des vagues, b) période moyenne des vagues, c) skewness et d) asymétrie verticale.

Le modèle représente bien l'évolution le long du profil bathymétrique des paramètres intégraux de l'état de mer, comme la hauteur significative $H_{m 0}$ (Figure 4a) et la période énergétique moyenne $T_{m-1,0}$ (Figure $4 \mathrm{~b}$ ) qui sont calculées à partir des moments du spectre de variance. Les effets des non-linéarités se traduisent par une augmentation de la hauteur significative avec la diminution de la profondeur d'eau due au shoaling et une diminution dans le creux du profil bathymétrique alors que la période moyenne 
commence par décroître quand l'énergie est transférée vers les plus hautes fréquences puis augmente lorsque le transfert s'inverse et persiste. D'autre part, deux paramètres permettant de caractériser l'asymétrie des vagues ont été analysés : la skewness (Figure 4c) et l'asymétrie verticale (Figure 4d), définies respectivement par :

$\lambda_{3}=\frac{\left\langle(\eta-\langle\eta\rangle)^{3}\right\rangle}{m_{o}^{3 / 2}}=\frac{\sum_{m=-\infty}^{+\infty} \sum_{n=-\infty}^{+\infty} \operatorname{Re}\left[B_{m, n}\right]}{m_{o}^{3 / 2}} \quad A=\frac{\sum_{m=-\infty}^{+\infty} \sum_{n=-\infty}^{+\infty} \operatorname{Im}\left[B_{m, n}\right]}{m_{o}^{3 / 2}}$

où $B_{m, n}=B\left(f_{m}, f_{n}\right)$ est le bispectre, défini comme la transformée de Fourier de la fonction d'auto-corrélation d'ordre 3 de l'élévation de surface libre. Ces deux paramètres rendent compte respectivement de l'asymétrie crête/creux et front/face arrière des vagues. Sur la partie plate avant le profil bathymétrique, où les vagues sont faiblement non-linéaires, ces paramètres ont des valeurs proches de zéro, mais évoluent ensuite avec le profil bathymétrique en bon accord avec les mesures, traduisant un développement de l'asymétrie des profils de vagues à la fois par rapport à un plan horizontal et par rapport à un plan vertical.

\section{Application et validation de la version 2DH du modèle numérique}

Ce cas test, basé sur les expériences de WHALIN (1971), met en œuvre une bathymétrie en forme de marche semi-circulaire qui joue le rôle d'une lentille convergente pour les vagues (Figure 5.a). Expérimentalement, des vagues régulières sont générées par un piston dans un bassin de 25,63 $\mathrm{m}$ de long et 6,096 $\mathrm{m}$ de large, dans une profondeur de 0,4572 m. Le profil bathymétrique utilisé dans la simulation est le même que celui utilisé par SHAO \& FALTINSEN (2014). Le cas simulé correspond à des vagues régulières de période $T=2 \mathrm{~s}$ et d'amplitude $a=0,0075 \mathrm{~m}$. Le potentiel des vitesses correspondant est imposé à la frontière gauche du modèle. Le domaine de calcul comprend la zone d'intérêt de $0 \mathrm{~m}$ à $25 \mathrm{~m}$ à laquelle on a ajouté une zone de relaxation de 3,91 m de large (une longueur d'onde) afin d'absorber les vagues réfléchies sur la marche et d'en diminuer la réflexion au niveau de la frontière gauche. Une zone de relaxation de 7,5 m de long (trois longueurs d'onde) a été ajoutée du côté de la frontière droite afin d'absorber les vagues. Les frontières latérales sont considérées comme des parois imperméables. Le domaine a été discrétisé par un nuage de points non-structuré de 60716 nœuds (approximativement espacés de 0,06 m). On simule une durée de $36 \mathrm{~s}$ (soit 18 périodes) avec un pas de temps constant $\Delta t=T / 75=0,0267 \mathrm{~s}$. Le nombre de nœuds par stencil est de $n=13$ et la constante de forme prise à $c=1$, après quelques tests de sensibilité des résultats aux valeurs de ces deux paramètres.

Le profil de la surface libre à l'instant final (figure 5.b) montre que, dans la partie de profondeur constante avant la marche, les vagues sont quasiment invariantes en $y$ alors qu'une fois passé la marche la structure du champ de vagues est tridimensionnelle. Une analyse harmonique des séries temporelles de surface libre suivant l'axe central du 


\section{XIV èmes Journées Nationales Génie Côtier - Génie Civil \\ Toulon, 29 juin au $1^{\text {er }}$ juillet 2016}

bassin permet d'obtenir l'évolution spatiale des amplitudes des trois premières harmoniques (Figure 6). La simulation donne des résultats en bon accord avec les résultats expérimentaux. La forte augmentation des amplitudes des harmoniques deux (2f) et trois (3f) avec la diminution de la profondeur d'eau est bien reproduite, montrant les capacités du modèle à simuler les effets de réfraction-shoaling non-linéaires sur une telle bathymétrie 3D avec effets de convergence très marqués.

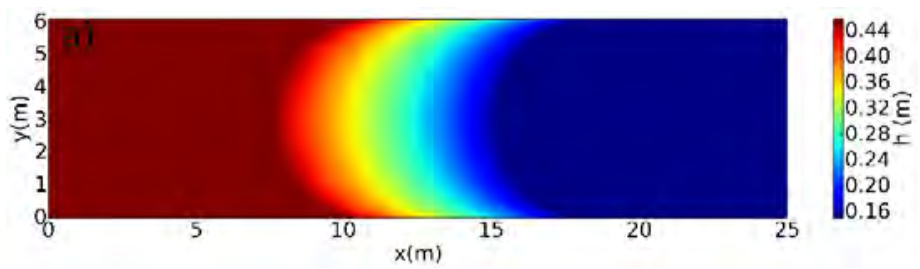

b)

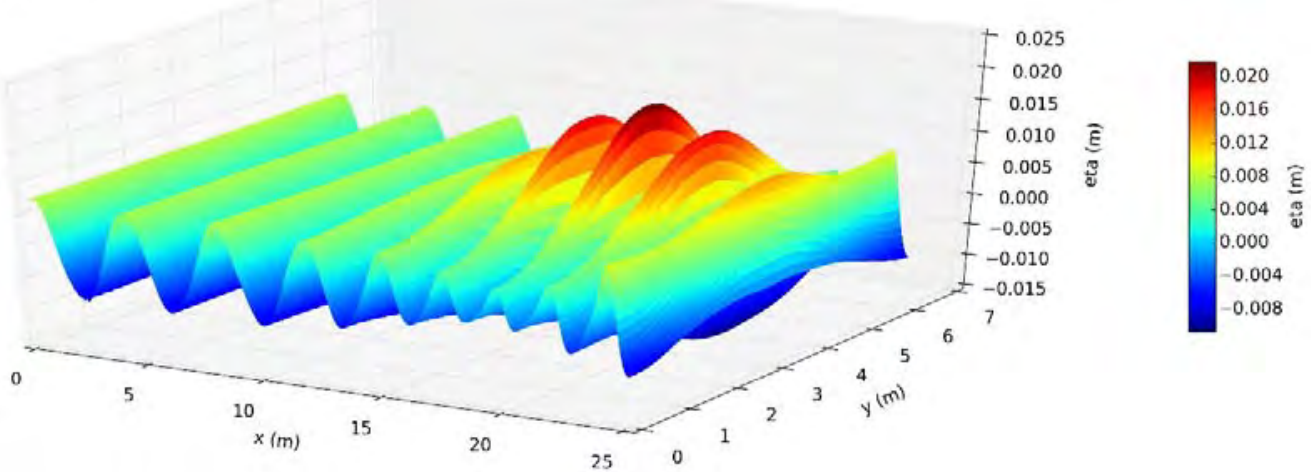

Figure 5. Bathymétrie (a) et position de la surface libre (b) simulée à l'instant final $(t \approx 36,045 s)$ pour l'expérience de WHALIN (1971).

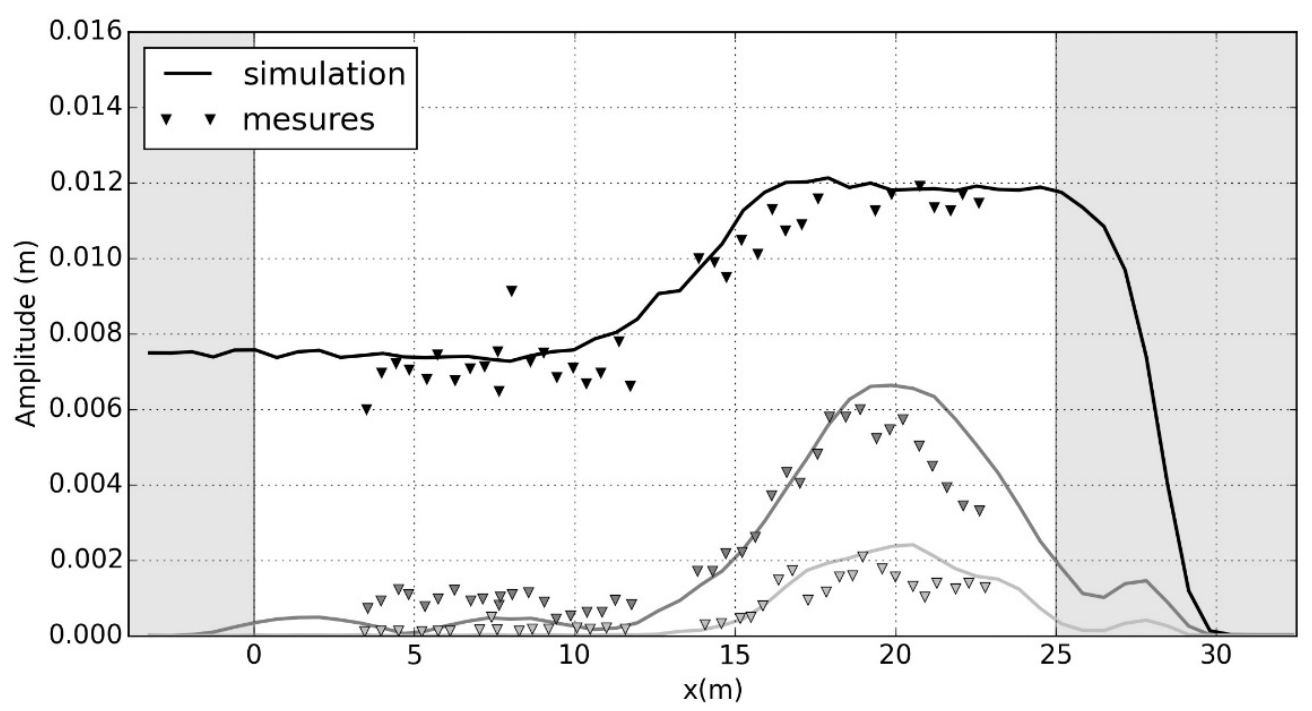

Figure 6. Evolution spatiale des amplitudes des trois premières harmoniques du cas test de WHALIN (1971). Les zones grisées correspondent aux zones de relaxation utilisées dans le modèle. 


\section{Conclusions et perspectives}

Le modèle numérique proposé, fondé sur une approche potentielle non-linéaire (équations surfaciques d'Euler-Zakharov), permet de modéliser les effets dispersifs et non-linéaires dans la transformation des vagues en zone côtière, sur une large gamme de profondeurs d'eau relatives. Les deux cas 1DH présentés ont permis de vérifier ces capacités dispersives et non-linéaires, notamment les transferts d'énergie entre les différentes harmoniques (libres ou liées) lors de leur génération et leur propagation. Les longueurs de battement des harmoniques 2 et 3 des expériences de CHAPALAIN et al. (1992) sont très bien restituées par le modèle. Lors de la transformation d'un spectre de vagues irrégulières sur un profil côtier réaliste (BECQ-GIRARD et al., 1999), nous avons montré un bon accord dans la dynamique d'évolution des pics harmoniques du spectre jusqu'à 5 fois la fréquence de pic.

De plus, l'utilisation d'une méthode utilisant des fonctions de bases radiales (RBF) pour l'extension du modèle à deux dimensions dans le plan horizontal a montré de bonnes performances quant à la représentation des effets non-linéaires sur le cas d'une des expériences 3D de WHALIN (1971) où une bathymétrie de forme semi-circulaire provoque une convergence de l'énergie des vagues vers l'axe du bassin par réfractionshoaling, avec à nouveau des effets non-linéaires importants. Un des avantages de cette méthode RBF est la possibilité de travailler sur des semis de points irréguliers, et donc d'avoir une résolution variable et adaptable en $(x, y)$ pour les applications réelles.

Outre les travaux en cours de tests et de validation de cette version 2DH du code Misthyc, les travaux futurs concernent la représentation de certains effets du déferlement et du run-up sur des talus inclinés. La version 2DH du modèle doit également être parallélisée en vue de son optimisation pour des applications d’ingénierie côtière et portuaire.

\section{Références bibliographiques}

BECQ-GIRARD F., FORGET P., BENOIT M. (1999) Non-linear propagation of unidirectional wave fields over varying topography. Coastal Engineering, Vol. 38, pp 91-113. http://dx.doi.org/10.1016/50378-3839(99)00043-5

BENOIT M., CHAZEL F. (2013). Validation expérimentale d'un modèle double-couche pour des vagues côtières non-linéaires et fortement dispersives. Revue Paralia, Vol. 6, pp 7.1-7.16. http://dx.doi.org/10.5150/revue-paralia.2013.007

BERKHOFF J.C.W. (1972) Computation of combined refraction-diffraction. Proceeding of the 13th International Conference on Coastal Engineering, ASCE, Vancouver (Canada), pp 470-490. 


\section{XIV ${ }^{\text {èmes }}$ Journées Nationales Génie Côtier - Génie Civil \\ Toulon, 29 juin au $1^{\text {er }}$ juillet 2016}

CHAPALAIN G., COINTE R., TEMPERVILLE A. (1992) Observed and modeled resonantly interacting progressive water-waves. Coastal Engineering, Vol. 16, pp 267300. http://dx.doi.org/10.1016/0378-3839(92)90045-V

CHAZEL F., LANNES D., MARCHE F. (2011). Numerical simulation of strongly nonlinear and dispersive waves using a Green-Naghdi model. Journal of Scientific Computing, Vol. 48(1-3), pp 105-116. http://dx.doi.org/10.1007/s10915-010-9395-9

KIRBY J.T. (2003). Boussinesq models and applications to nearshore wave propagation, surf zone processes and wave-induced currents. Advances in Coastal Modeling, V.C. Lakhan (ed), Elsevier, pp 1-41.

MADSEN P.A., MURRAY R., SORENSEN O.R. (1991). A new form of the Boussinesq equations with improved linear dispersion characteristics. Coastal Engineering, Vol. 15, pp 371-388. http://dx.doi.org/10.1016/0378-3839(91)90017-B

MADSEN P.A., BINGHAM H.B., LIU H. (2002). A new Boussinesq method for fully nonlinear waves from shallow to deep water. Journal of Fluid Mechanics, Vol. 462, pp 1-30. http://dx.doi.org/10.1017/S0022112002008467

MADSEN P.A., FUHRMAN D.R., WANG B. (2006). A Boussinesq-type method for fully nonlinear waves interacting with a rapidly varying bathymetry. Coastal Engineering, Vol. 53, pp 487-504. http://dx.doi.org/10.1016/j.coastaleng.2005.11.002

NWOGU O.G. (1993). Alternative form of Boussinesq equations for nearshore wave propagation. Journal of Waterway, Port, Coastal and Ocean Engineering, Vol. 119, pp 618-638. http://dx.doi.org/10.1061/(ASCE)0733-950X(1993)119:6(618)

RAOULT C., BENOIT M., YATES M.L. (2014) Etude et qualification d'un modèle numérique complètement non-linéaire et dispersif pour les vagues en zone côtière. XIIIèmes Journées Nationales Génie Côtier Génie Civil, 2-4 juillet 2014, Dunkerque (France), pp 159-168. http://dx.doi.org/10.5150/jngcgc.2014.018

SHAO Y-L., FALTINSEN O.M. (2014) A harmonic polynomial cell (HPC) method for $3 D$ Laplace equation with application in marine hydrodynamics. Journal of Computational Physics, Vol. 274, pp 312-332. http://dx.doi.org/10.1016/j.jcp.2014.06.021

TIAN Y., SATO S. (2008). A numerical model on the interaction between nearshore nonlinear waves and strong currents. Coastal Engineering Journal, Vol. 50(4), pp 369395. http://dx.doi.org/10.1142/S0578563408001879

ZAKHAROV V.E. (1968). Stability of periodic waves of finite amplitude on the surface of a deep fluid. Journal of Applied Mechanics and Technical Physics, Vol. 9(2), pp 190-194. http://dx.doi.org/10.1007/BF00913182

WRIGHT G.B., FORNBERG B. (2006) Scattered node compact finite difference-type formulas generated from radial basis functions. Journal of Computational Physics, Vol. 212, pp 99-123. http://dx.doi.org/10.1016/j.jcp.2005.05.030

WHALIN R.W. (1971) The limit of applicability of linear wave refraction theory in a convergence zone. Technical report, DTIC Documents. 
Thème 1 - Hydrodynamique côtière

YATES M.L., BENOIT M. (2015) Accuracy and efficiency of two numerical methods of solving the potential flow problem for highly nonlinear and dispersive water waves. International Journal for Numerical Methods in Fluids. Vol. 77(10), pp 616-640. http://dx.doi.org/10.1002/fld.3992 